\title{
Study on the Mechanism of Mulberry Root Bark Decoction in the Treatment of Chronic Obstructive Pulmonary Disease based on Network Pharmacology
}

\author{
Jie Meng1, Xuanguo Zhang2* \\ ${ }^{1}$ Shaanxi University of Chinese Medicine, Xianyang 712046, Shaanxi Province, China \\ ${ }^{2}$ Shaanxi Provincial Hospital of Chinese Medicine, Xi'an 710000, Shaanxi Province, China \\ *Corresponding author: Xuanguo Zhang, 121269700@qq.com
}

\begin{abstract}
Objective: Study the mechanism of Mulberry Root Bark Decoction in the treatment of COPD based on network pharmacology. Methods: The active components and predictive targets of Mulberry Root Bark Decoction were screened by TCMSP database. The disease targets of COPD were collected by searching GeneCards, DisGeNET, PhamGKB and TTD databases. Using R language to draw Venn diagram, and get the key target of Mulberry Root Bark Decoction in the treatment of COPD. Cytoscape was used to construct the regulatory network of drug active ingredient disease target. The key targets were imported into string database to construct protein-protein interaction network, and the core targets were obtained by network topology analysis with Cytoscape software. Finally, the Bioconductor platform and R language were used for GO and KEGG enrichment analysis. Results: There were 142 active components and 255 drug targets in Mulberry Root Bark Decoction. 1941 COPD targets were retrieved. There were 129 common targets of Mulberry Root Bark Decoction and COPD; Eight core targets of PPI network were obtained. GO function analysis is involved in oxidative stress, cellular chemical stress and other biological processes. Cell components such as cell membrane raft and membrane region involve molecular functions such as ubiquitin like protein ligase and DNA binding transcription factor. KEGG mainly includes PI3K-Akt signal pathway, tumor necrosis factor signal pathway, IL-17 signal pathway, etc. Conclusion: Quercetin, luteolin, kaempferol, wogonin and other active components in Mulberry Root Bark Decoction act on PI3K / Akt, TNF, IL-17, TCR and other signal pathways through Jun, TP53, MAPK1, IL6 and other targets to play an anti-inflammatory and reduce oxidative stress response role. The results of this study can provide a reference for further study on the mechanism of Mulberry Root Bark Decoction in the treatment of chronic obstructive pulmonary disease.
\end{abstract}

Keywords: Mulberry Root Bark Decoction; Network pharmacology; COPD; Action mechanism

Publication date: September 2021; Online publication: September 30, 2021

\section{Introduction}

COPD belongs to the category of "lung distention" in traditional Chinese medicine. COPD is mainly characterized by persistent airflow restriction, and its clinical manifestations are cough, chest tightness, asthma and so on. At present, COPD is the fourth leading cause of human death. The pathogenesis of the disease remains to be clarified. Studies have shown that it is closely related to inflammation and oxidative stress ${ }^{[1]}$. Glucocorticoids and bronchodilators are commonly used to treat the disease clinically, but longterm use is easy to produce drug resistance and toxic and side effects on the body. Therefore, it is of great significance to find safe and effective traditional Chinese medicine for the treatment of COPD. Mulberry 
Root Bark Decoction comes from the Encyclopedia of Ancient and Modern Medicine System. It main used to treat the symptom of lung Qi excess, phlegm fire exuberant and feeling short of breath. Modern traditional Chinese medicine is mostly used to treat COPD. According to clinical observation, Mulberry Root Bark Decoction can effectively improve its clinical symptoms, arterial blood gas indexes and pulmonary function ${ }^{[2]}$. At present, the mechanism of Mulberry Root Bark Decoction in the treatment of COPD is not clear, and there is a lack of experimental research. Therefore, this study uses the method of network pharmacology to explore the specific molecular mechanism of Mulberry Root Bark Decoction in the treatment of COPD from a micro perspective through data collection, sorting and analysis.

\section{Methods and results}

\subsection{Screening of components and potential targets of Mulberry Root Bark Decoction}

The chemical constituents and action targets of Mulberry Root Bark Decoction, such as Cortex Mori, Rhizoma Pinelliae, Fructus Perillae, bitter apricot kernel, Bulbus Fritillariae, Fructus Gardeniae, Radix Scutellariae and Coptis chinensis were searched by TCMSP database. According to the pharmacokinetic (ADME) parameters, the oral bioavailability $(\mathrm{OB} \geq 30 \%)$ and drug like index ( $\mathrm{DL} \geq 0.18$ ) were set as the screening conditions to obtain the effective active components and potential targets of Mulberry Root Bark Decoction. UniProt database standardized the obtained target proteins, and finally obtained 157 active components and 1944 targets of Mulberry Root Bark Decoction.

\subsection{COPD disease target acquisition}

Taking "chronic obstructive pulmonary disease" as the keyword, 2117 targets were obtained by searching genecards, PharmGKB, TTD and disgenet databases. After the above targets were de duplicated, 1941 targets of COPD were obtained.

\subsection{Network construction of active components and key targets of Mulberry Root Bark Decoction in the treatment of COPD}

The potential targets of active components of Mulberry Root Bark Decoction were matched with COPD disease targets by R language, and 129 common targets were obtained. It is introduced into Cytoscape to construct the regulation network of "active ingredient - key target". The network shows that there are 227 nodes and 729 edges. Among them, PTGS2, PTGS1, AR and NOS2 are the target genes with the top four connectivity values, and quercetin, luteolin, kaempferol and wogonin are the drug active components with the top four connectivity values. See Figure 1.

\subsection{Construction of protein interaction network (PPI) of Mulberry Root Bark Decoction in the treatment of COPD}

The common targets of Mulberry Root Bark Decoction and COPD were imported into string database to obtain the protein-protein interaction relationship. Import it into Cytoscape software, score the network with CytoNCA plug-in, take the value greater than the median value as the screening condition, further obtain the core protein interaction relationship, and construct the PPI core network, as shown in Figure 2. Finally, 8 PPI network core proteins were obtained, which were JU, TP53, MAPK1, IL6, TNF, RELA, MYC and IL2.

\subsection{GO and KEGG enrichment analysis}

Go $(\mathrm{P}<0.05)$ and KEGG enrichment analysis $(\mathrm{P}<0.05)$ were performed on the core targets of Mulberry Root Bark Decoction in the treatment of COPD through R language and Bioconductor platform, as shown in Figure 3. and 4. Go analysis items show the biological processes which include oxidative stress, cell 
chemical stress, etc. Molecular function is related with ubiquitin like protein ligase, DNA binding transcription factor and so on. Cell components mainly involve cell membrane raft, membrane micro region and so on. KEGG mainly involves signal pathways such as PI3K / Akt, TNF, IL-17 and TCR.

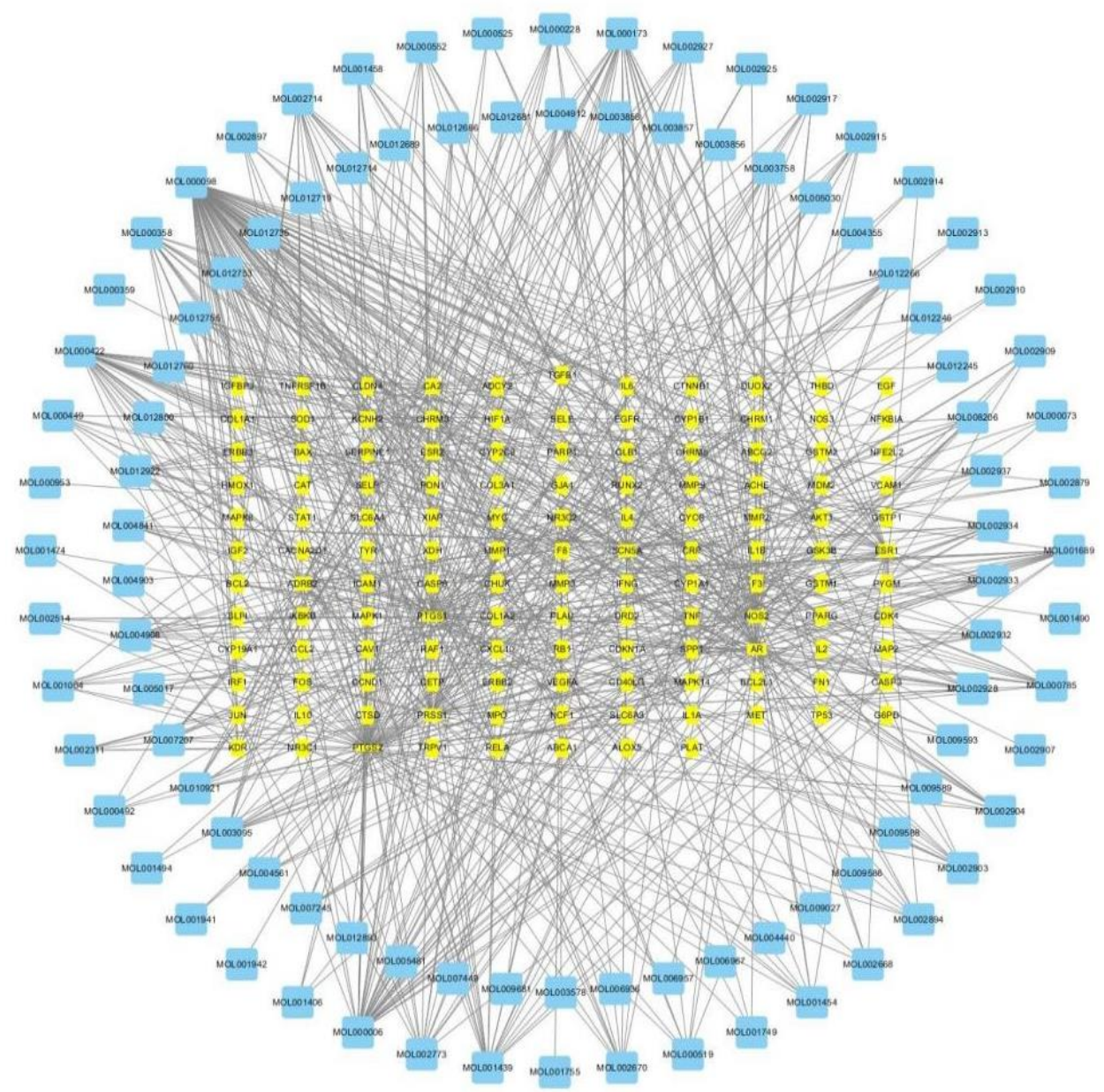

Figure 1. Regulation network of active components - key targets of traditional Chinese Medicine
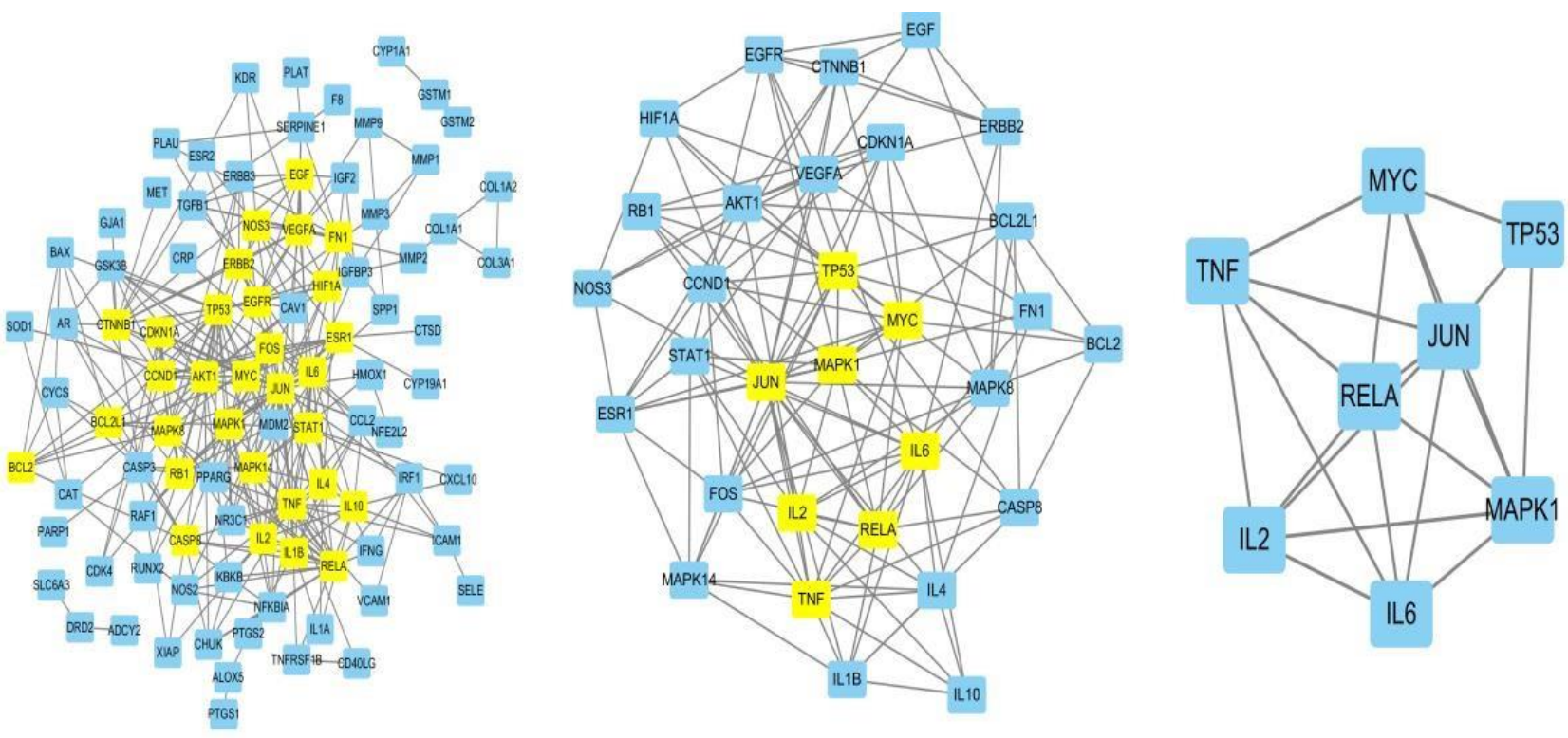

Figure 2. PPI core protein interaction 


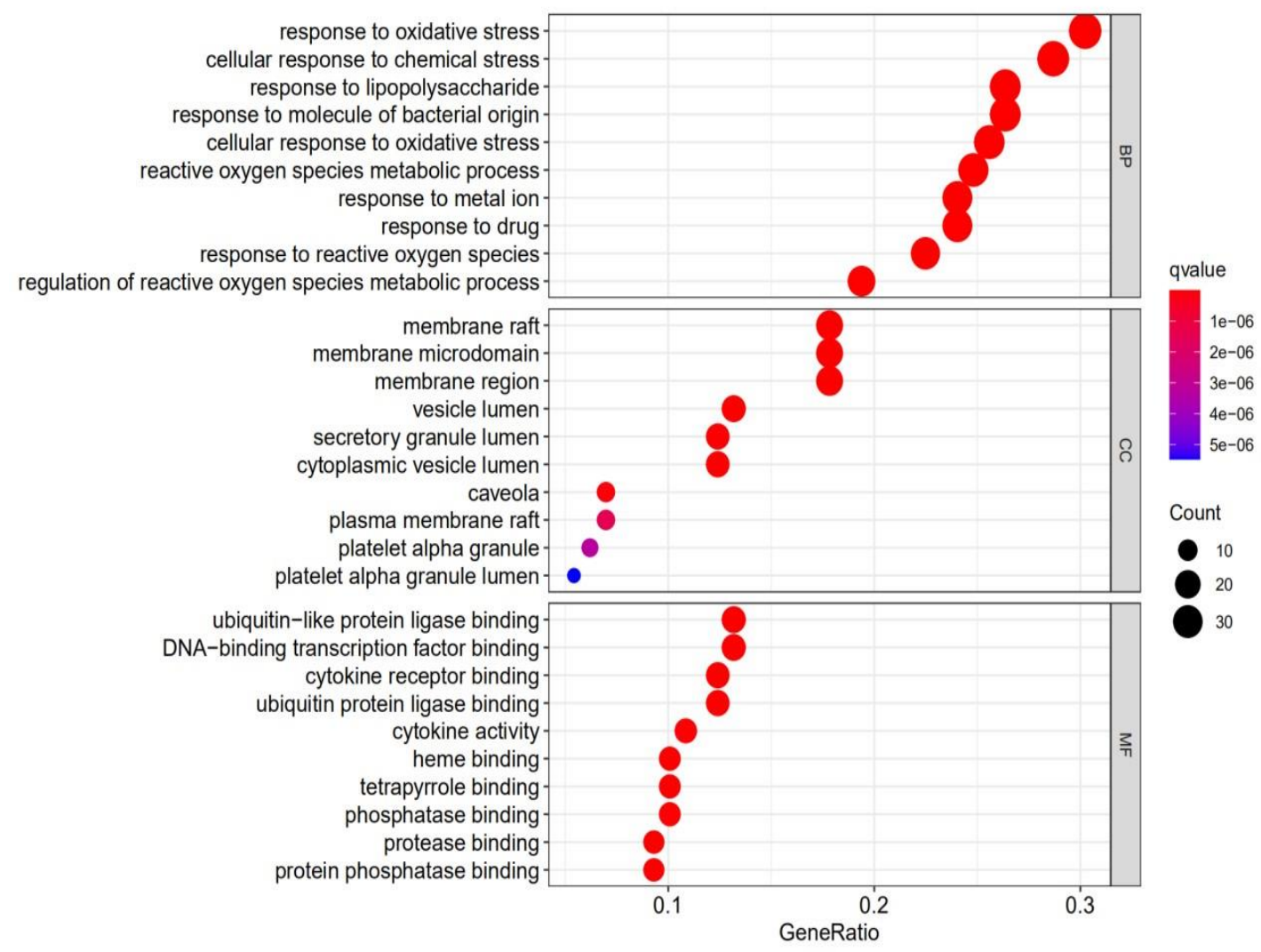

Figure 3. Bubble diagram of go function analysis

Fluid shear stress and atherosclerosis PI3K-Akt signaling pathway AGE-RAGE signaling pathway in diabetic complications

herpesvirus infection Human cytomegalovirus infection Hepatitis C TNF signaling pathway Hepatitis B Epstein-Barr virus infection Prostate cancer Human T-cell leukemia virus 1 infection IL-17 signaling pathway Endocrine resistance Toxoplasmosis Hepatocellular carcinoma Influenza A Chagas disease Relaxin signaling pathway Apoptosis

Pancreatic cancer Small cell lung cancer C-type lectin receptor signaling pathway Colorectal cancer T cell receptor signaling pathway Chronic myeloid leukemia Leishmaniasis Bladder cancer Platinum drug resistance

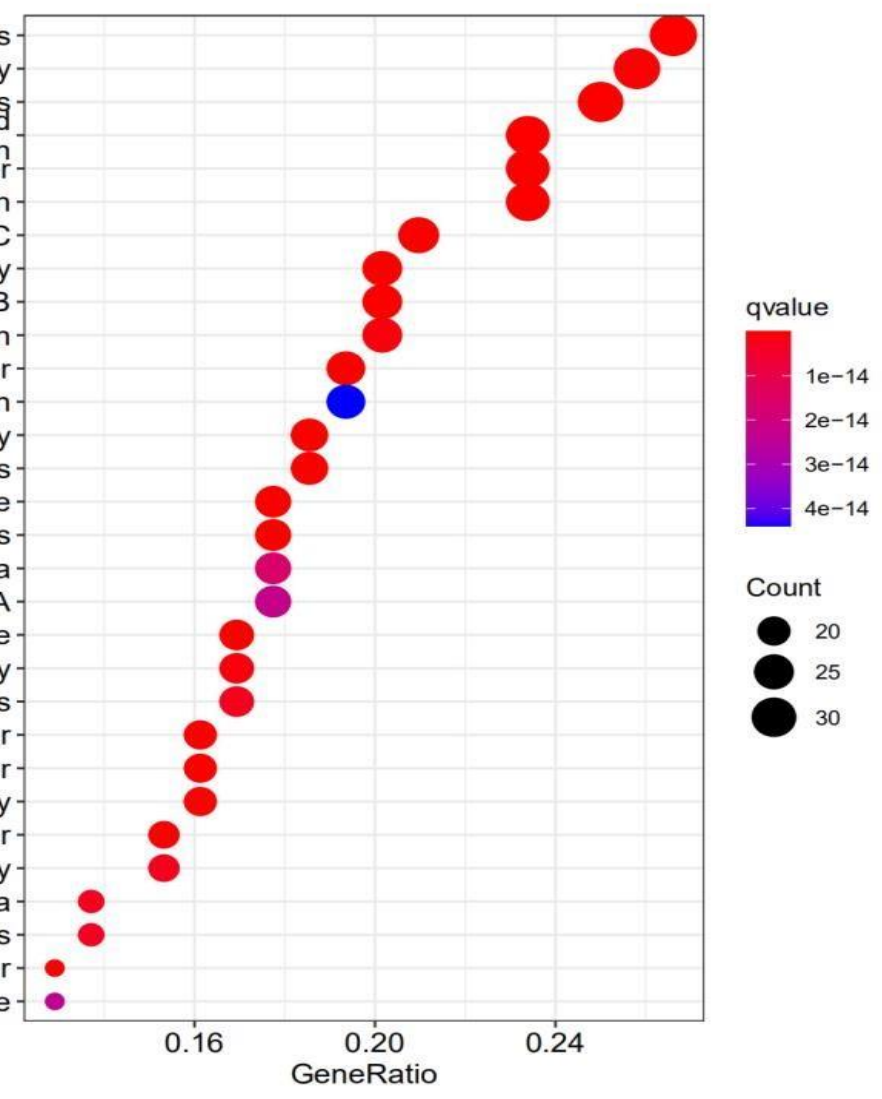

Figure 4. Bubble Diagram of KEGG enrichment pathway 


\section{Discussion}

The results showed that the main active components of Mulberry Root Bark Decoction were quercetin, luteolin, kaempferol, wogonin and so on. Studies have shown that quercetin can down regulate proinflammatory factors IL6 and TNF- $\alpha$, up regulate anti-inflammatory factor IL10, as well as reduce inflammatory factor and reduce TGF- $\beta 1$ and $\alpha$ - SMA to inhibit apoptosis. At the same time, it can reduce the aggregation of neutrophils, CD11b + / CD11c + macrophages and CD8 + T cells in the lungs of COPD mice, so as to play a therapeutic role ${ }^{[3]}$. Luteolin significantly inhibited the expression of TNF- $\alpha$ and IL$10 \mathrm{mRNA}$ in lung tissue, which play a significant role in anti-oxidation and anti-inflammatory ${ }^{[4]}$. Kaempferol inhibits TNF- $\alpha$ and IL- 6 levels and NF- $\kappa$ B activation to reduce oxidative stress response and inflammation-induced injury ${ }^{[5]}$. Wogonin could significantly inhibit the inflammatory response of macrophage RAW264.7, down regulate the level of intracellular ROS and extracellular TNF- $\alpha$, and play anti-inflammatory and antioxidant role ${ }^{[6]}$.

PPI core protein interaction diagram showed that the active components of Mulberry Root Bark Decoction acted through JUN, TP53, MAPK1, IL6, TNF, RELA, MYC, IL2 and other key targets. JUN regulates inflammation by targeting IL- $1 \beta$ synthesis and macrophage activation ${ }^{[7]}$. Activation of MAPK1 can release cytokines such as TNF- $\alpha$ and IL-6, which play a key role in the expression of proinflammatory factor ${ }^{[8]}$. IL-6 is mainly produced by monocyte macrophages, Th2 cells, vascular endothelial cells, etc., which can activate $\mathrm{T}$ cells, differentiate and proliferate as well as participate in inflammatory response ${ }^{[9]}$. TNF can improve the phagocytosis of neutrophils and promote the adhesion of neutrophils to endothelial cells, thus stimulating the body to produce an inflammatory response.

The results of pathway enrichment showed that PI3K / Akt, IL-17 and other signal pathways were the main pathway of Mulberry Root Bark Decoction in the treatment of COPD. PI3K Akt can reduce the activation of NLRP3 and IL-18 and reduce airway inflammation in COPD by inhibiting the expression of ASK1 and TSC1 in this pathway ${ }^{[10]}$. IL-17 signaling pathway is involved in inflammation and immune system diseases. Helper T cells 17 recruit and activate neutrophils in the airway by secreting IL-17 and IL23 , so as to involve in the inflammatory response.

In summary, this study analyzed the active ingredients, key targets and main pathways of Mulberry Root Bark Decoction in the treatment of COPD through network pharmacology. It was found that quercetin, luteolin, kaoneferol, baicalin and so on in Mulberry Root Bark Decoction play an anti-inflammatory role and reduce oxidative stress response through JUN, TP53, MAPK1, IL6 and TNF and other target spot on PI3K/Akt, TNF, IL-17 and other signaling pathways. Traditional Chinese medicine compound has the characteristics of multi-component and multi-target. This study only discusses the molecular mechanism of treatment from the micro perspective, and its specific mechanism needs to be further verified by experiments.

\section{Disclosure statement}

The author declares no conflict of interest.

\section{References}

[1] Ma Y, Han LH. 2020, Clinical Study on Reduening Injection Combined with Compound Ipratropium Bromide in the Treatment of Acute Exacerbation of Chronic Obstructive Pulmonary Disease. Drugs \& Clinic, (12): 2430-2435.

[2] Fan JJ, Li G, Shi HL, 2019, Observation on the Efficacy of Modified Mulberry Root Bark Decoction in the Treatment of Acute Exacerbation of Chronic Obstructive Pulmonary Disease. China Medicine and Pharmacy, (03): 17-20+32. 
[3] Mohammad F, Mishra R, Jing Y, et al., 2018, Quercetin Prevents Rhinovirus-Induced Progression of Lung Disease in Mice with COPD Phenotype. Plos One, 13(7).

[4] Celebi D, et al. 2020, "Protective effect of luteolin on acute lung injury in a rat model of sepsis." Biotechnic and Histochemistry. 1-7.

[5] Yao H, Sun J, Wei J, et al., 2020, Kaempferol Protects Blood Vessels From Damage Induced by Oxidative Stress and Inflammation in Association With the Nrf2/HO-1 Signaling Pathway. Frontiers in Pharmacology, 11(11).

[6] Zhou SY, You LM, Zhang HL, 2020, Inhibitory Effect of wogonin on LPS-ATP-Induced Macrophage Oxygenation Stress. Chinese Journal of Immunology, (03): 257-260+266.

[7] Diaz-Cañestro C, et al., 2019, AP-1 (Activated Protein-1) Transcription Factor JunD Regulates Ischemia/Reperfusion Brain Damage via IL-1 $\beta$ (Interleukin-1 $\beta$ ). Stroke, 50(2).

[8] Zhang XH, Li FF, Qi Y, et al., 2020, Effects of Electroacupuncture on the Expression of Mitogen Activated Protein Kinase and Cytokines in Peritoneal Mast Cells of Urticaria Rats. Acupuncture Research, (04): 299-304.

[9] Yang WY, et al., 2020, Correlation between Serum IL-6, CRP, Plasma NT-probNP and Lung Function in COPD Patients and Its Predictive Value to Disease. Laboratory Medicine and Clinic, (23): 3459-3462.

[10] Chen ZR, et al., 2020, The Role of PI3K Signaling Pathway in Airway and Systemic Inflammation in Rats with Chronic Obstructive Pulmonary Disease. Chinese Journal of Gerontology, (23): 50325035 . 